\title{
P 043 OUT-OF-HOURS PALLIATIVE CARE: WHAT ARE THE EDUCATIONAL NEEDS AND PREFERENCES OF GENERAL PRACTITIONERS?
}

Claire Magee, ${ }^{1,2}$ Jonathan Koffman'. 'Marie Curie Hospice West Midlands, Solihull, UK; ${ }^{2}$ King's College London, UK

\subsection{6/bmjspcare-2014-000654.84}

Background General practitioners (GPs) provide most out-of-hours medical care, but overall see few palliative patients. Keeping their knowledge and skills up-to-date is challenging. UK national policy has highlighted the importance of improving training for out-of-hours clinicians in order to reduce unnecessary admissions and support patients to die at home. However, little is known about the educational preferences of GPs working in this setting.

Aims To explore the confidence of out-of-hours GPs in symptom control, and to identify their educational needs and preferences in order to inform recommendations for future educational programmes.

Methods A structured postal survey was designed and sent to 1005 GPs employed by an independent provider of out-of-hours services across England. Quantitative data was analysed using descriptive statistics and non-parametric tests.

Results 203 (20.3\%) GPs responded to the survey questionnaire. $13.3 \% \quad(n=27)$ worked exclusively out-of-hours; they were typically older and more experienced. Confidence in assessing palliative care emergencies $(42.8 \%, \mathrm{n}=87$ : 'not so confident' or 'not at all confident'), managing symptoms in non-cancer patients $(39.4 \%, \mathrm{n}=80)$ and prescribing a new syringe driver $(39.0 \%, \mathrm{n}=79)$ was lowest. Low confidence was associated with infrequent exposure $(\mathrm{p}<0.05)$ and lack of training $(\mathrm{p}<0.05) .12 .8 \%(\mathrm{n}=26)$ had never received formal palliative care training. Educational preferences were closely associated with confidence $(\mathrm{p}<0.0005)$; the above topics were most requested. E-learning was the preferred method $(67.5 \%$, $\mathrm{n}=137)$. GPs were more likely to prefer education with other GPs than with other professionals $(p=0.001) .82 .1 \%(n=165)$ felt training focused on out-of-hours work would be beneficial.

Conclusions Confidence in key palliative care competences is lacking. Educational strategies must be targeted at GPs needs, both in terms of content and delivery, with regular updates. E-learning is favoured, but should be combined with other approaches such as out-of-hours themed workshops. Specialist palliative care services should engage with out-of-hours providers to support education. 